\title{
A comparison of ground geoelectric activity between three regions of different level of seismicity
}

\author{
A. Ramírez-Rojas ${ }^{1}$, E. L. Flores-Márquez ${ }^{1,2}$, L. Guzmán-Vargas ${ }^{4}$, J. Márquez-Cruz ${ }^{2}$, C. G. Pavía-Miller ${ }^{1}$, and \\ F. Angulo-Brown ${ }^{3}$ \\ ${ }^{1}$ Área de Física de Procesos Irreversibles, Universidad Autónoma Metropolitana Azcapotzalco, Avenida San Pablo 180 \\ Colonia Reynosa, Azcapotzalco, 02200, México D.F., México \\ ${ }^{2}$ Instituto de Geofísica, UNAM, Circuito Exterior S/N, Cd. Universitaria, 04510 México D.F., México \\ ${ }^{3}$ Departamento de Fisica, Escuela Superior de Fisica y Matemáticas, Instituto Politécnico Nacional, Edif. 9, U.P. Zacatenco, \\ 07738, México D.F., México \\ ${ }^{4}$ Unidad Profesional Interdisciplinaria en Ingenieria y Tecnología Avanzadas, Instituto Politécnico Nacional, 07738, México \\ D.F., México
}

Received: 24 July 2007 - Revised: 4 October 2007 - Accepted: 5 October 2007 - Published: 16 October 2007

\begin{abstract}
In this work, we present a statistical study of geoelectric time series from three Mexican regions with recognized different levels of seismicity. This study is made by means of both the Higuchi's method and the detrended fluctuation analysis for the detection of fractal behavior. With these methods we present scatter plots corresponding to scaling exponents for short and large lags arisen from crossover points in the geoelectric data. Through these scatter plots we observe a reasonable segregation of clouds of points corresponding to the three mentioned regions. These results permit to suggest that a different level of characteristic seismicity in one region is translated into a different level of geoelectric activity.
\end{abstract}

\section{Introduction}

Since some decades ago the possible correlation between patterns of electric self-potential of the ground and the preparation mechanism of earthquakes (EQ) have been investigated with the aim of searching for possible precursory signatures of EQ occurrence (Varotsos and Alexopodus, 1984a, 1984b). This investigation has continued until the present day without conclusive results (Telesca et al., 2006, 2007; Gotoh et al., 2003). Nevertheless the complexity of this problem, there are no reasons yet for abandoning this line of investigation. Searching for 1 to 1 correspondence between anomalies in electromagnetic signals and earthquakes with relatively large magnitude was the main goal in the stud-

Correspondence to: A. Ramírez-Rojas

(arr@correo.azc.uam.mx) ies in this field (Varotsos et al., 1984a; Ramírez-Rojas et al., 2004a, b; Telesca et al., 2003; Hayakawa et al, 1999; Hayakawa and Ito, 2000). The analysis techniques of anomalous electromagnetic patterns have evolved from visual inspection (Varotsos and Alexopodus, 1984a, b) until more sophisticated approaches such as fractal and multifractal methods, and others stemming from chaos theory and nonlinear dynamics (Varotsos et al., 2002; Varotsos et al., 2003a, b; Nikolopoulos et al., 2004; Eftaxias et al., 2004; Abe, 2005). For example, Smirnova et al. (2001) and Gotoh et al. (2003) used spectral and fractal analyses to investigate ULF geomagnetic data associated to EQs at Guam and Izu peninsula, respectively. Telesca et al. (2001, 2005a, b) investigated EQs at southern Italy by means of Hurst exponent and multifractal spectra of geoelectric signals. On the other hand, the generation of transient electric potential prior to rupture has been also demonstrated in a number of laboratory experiments involving both dry and wet rock specimens (Villianatos et al., 2004; Freund, 2002; Sobolev, 2004). Thus, in principle, there are evidences from laboratory to field observations about the possible link between electromagnetic anomalous signals and the mechanisms of rock fracture. However, nowadays all this phenomenology lacks of a solid theoretical basis regardless of interesting proposals such as electrokinetic effect (Dobrovolsky et al., 1989), pressurestimulated currents (Villianatos et al., 2004) and other theoretical models (Stacey, 1964; Stacey and Johnson, 1972; Nitsan, 1977). In the present article we perform a comparative study of the global behavior of seismicity and geoelectric activity in three Mexican regions of different level of seismicity (see Fig. 1) linked to the Middle American trench, which is the border between the Cocos and the American tectonic

Published by Copernicus Publications on behalf of the European Geosciences Union. 


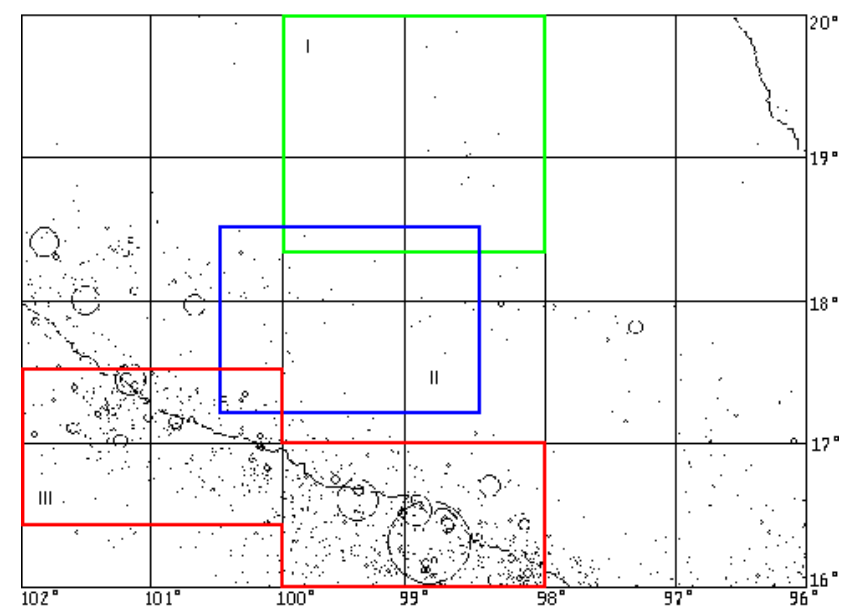

Fig. 1. Map of the three Mexican seismic regions considered. Region I of low seismicity level, II with medium seismicity level, and III with high seismicity level.

plates. The regions considered in this paper are constituted of composite terrains with both undersea volcanic and sedimentary sequences (Ramirez-Guzman, 1996; Angulo-Brown et al., 1998). Our approach is global in the sense that we do not search for correlations between extreme events, but we only observe the general behavior of geoelectric activity of the three regions by means of fractal dimensions and detrended fluctuation analysis. Our results suggest that regions of different seismicity also have global different geoelectric behavior independently of singular events, that is, seemingly the global differences between stress fields, tectonic structures and crust features are expressed as global differences in geoelectric activity. The paper is organized as follows: In Sects. 1 and 2, we present the methodology used, in Sect. 3 we introduce the method of data processing; in Sect. 4 the results of the application of the methods of analysis are showed and finally in Sect. 5 the discussion and concluding remarks are presented.

\section{Methodology: Higuchi's dimension and DFA}

First, we provide an outline of the fractal technique developed by Higuchi (1988). This method gives stable indices even for a small number of data. Higuchi $(1988,1990)$ considers a finite set of time series of length $\mathrm{N}$, taken at a regular interval:

$x(1), x(2), x(3), \cdots \cdots, x(N)$

From the given time series, a new time series, $x_{k}^{m}$ is obtained and defined as follows

$x_{k}^{m}: x(m), x(m+k), x(m+2 k), \ldots, x\left(m+\left[\frac{N-k}{k}\right] k\right)$

where $m=1,2, \ldots, k$, and [ ] denotes the Gauss's notation. Here $m$ and $k$ are integers that indicate the initial time and
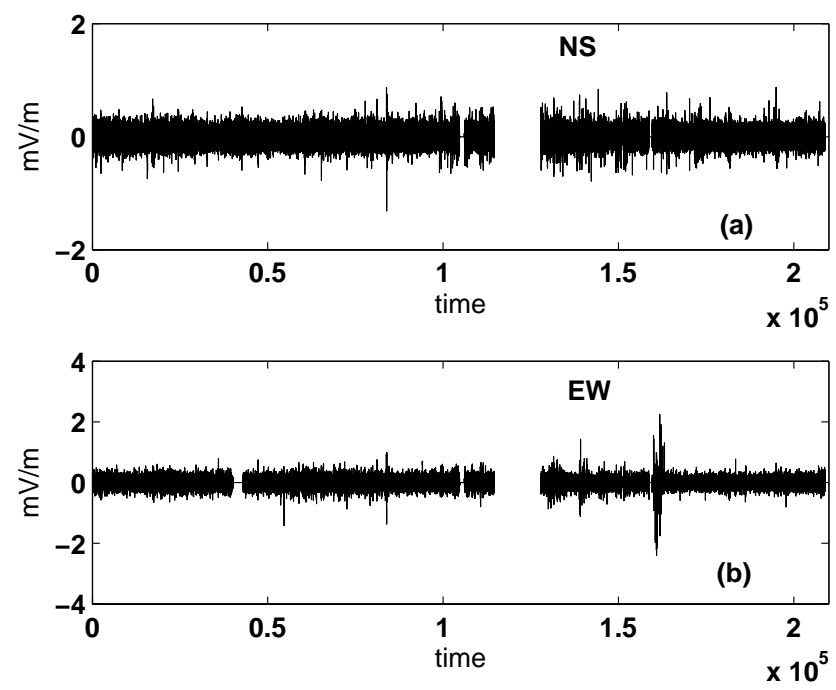

Fig. 2. Pre-processed time series of Acapulco station, 1993. (a) NS channel, (b) EW channel.

the time interval respectively. For a time interval equal to $k$, one gets $k$ sets of new time series. Higuchi defines (1988) the length of the curve associated to each time series $x_{k}^{m}$ as follows:

$L_{m}^{k}=\sum_{i=1}^{\left[\frac{N-m}{k}\right]}[x(m+i k)-x(m+(i-1) k)]\left(\frac{N-1}{\left[\frac{N-m}{k}\right] k}\right) \frac{1}{k}$

where the term $\frac{(N-1)}{\left[\frac{N-m}{k}\right] k}$ is a normalization factor. The length of the curve for the time interval $k$ is taken as the average value $\langle L(k)\rangle$ over $k$ sets of $L_{m}(k)$. If the average value obeys the scaling law:

$\langle L(k)\rangle \propto k^{-D}$

then the curve is fractal with dimension $D$ (Higuchi, 1988). This algorithm can be applied even to non-stationary time series and this fact represents an advantage over the spectral techniques (Cervantes de la Torre et al., 1999). The fractal dimension $D$, taken from Eq. (2) is calculated as the slope of the double log plot of $\langle L(k)\rangle$ against $k$. Applying the Higuchi's algorithm to data as those of Figs. 2, 3, and 4, stable scaling exponents are obtained.

The second technique that we applied was introduced by Peng et al. (1994, 1995), and it consists of a modified root mean square analysis of a random walk termed detrended fluctuation analysis (DFA). The DFA has advantages over conventional methods because it permits the detection of long-range correlations embedded in a seemingly non stationary time series, and also avoids the spurious detection of apparent long-range correlations that are an artifact of nonstationarity. Briefly the DFA algorithm is described as follows: Consider a time series $x(1), x(2), x(3), \cdots \cdots, x(N)$. 

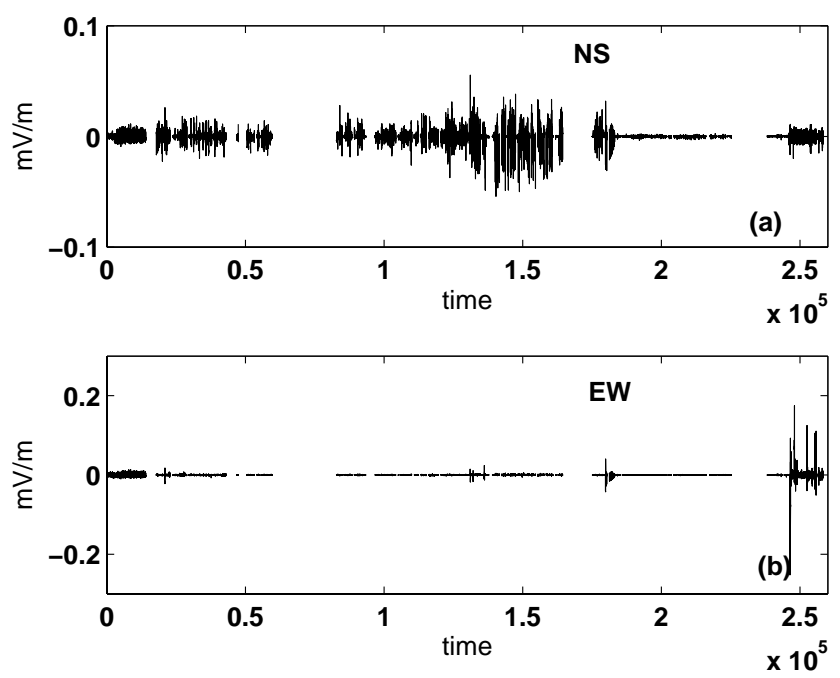

Fig. 3. Pre-processed time series of Chilpancingo station, 1993, (a) NS channel, (b) EW channel.

A new time series $\{y(k)\}$ is obtained by integration of the original time series, $y(k)=\sum_{i=1}^{k}\left(x(i)-x_{\text {ave }}\right)$ where $x_{\text {ave }}$ is the average of the data set. Next, the integrated time series is divided into boxes of equal length $n$. For each box of length $n$, a least-squares line is fitted to the data, (representing the trend in each box: $\left.y_{n}(k)\right)$. Next, the integrated time series is detrended by subtracting $y(k)-y_{n}(k)$ in each box. The root mean-square fluctuation of this integrated and detrended time series is calculated by

$F(n)=\sqrt{\frac{1}{N} \sum_{k=1}^{N}\left[y(k)-y_{n}(k)\right]^{2}}$.

This computation is repeated over many time scales (box sizes) to provide a relationship between $F(n)$, and the box size $n$. Typically $F(n)$ will increase with box size $n$. A linear relationship on a double log graph indicates the presence of scaling, that is:

$F(n) \propto n^{\alpha}$

The value of the scaling exponent $\alpha$, characterizes the correlation in a time series. For example white noise has $\alpha=0.5$. Two special cases are $\alpha=1$ corresponding to a $1 / f$ noise and $\alpha=1.5$ to a Brownian noise. Values into the interval $0.5<\alpha \leq 1$, indicate persistent long-range power-law correlations. In contrast, $0<\alpha<0.5$ indicates a different type of power-law correlation such that large and small values of the time series are more likely to alternate.

\section{Data processing}

Electric self-potential data consist of the measurements of potential differences, $\Delta \mathrm{V}$, between two electrodes buried $2 \mathrm{~m}$
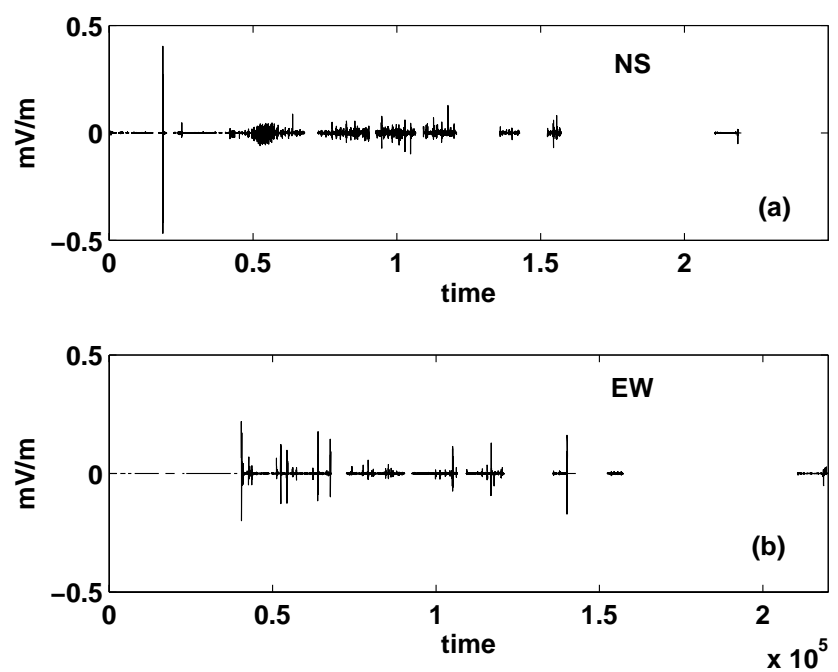

Fig. 4. Pre-processed time series of Puebla station, 1992. (a) NS channel, (b) EW channel.

of depth into the ground and separated $50 \mathrm{~m}$ of distance. A couple of electrodes was oriented in North-South direction, NS channel, and other couple in East-West direction, EW channel (Yepez et al., 1995), as it indicates VAN methodology (Varotsos and Alexopodus, 1984a, b). This survey project has consisted in six monitoring stations and had been in operation from 1992 to 1996 . The data considered in this study correspond to three monitoring stations located at the cities of Acapulco (A) $\left(16.85^{\circ} \mathrm{N}, 99.9^{\circ} \mathrm{W}\right)$, Chilpancingo (C) $\left(17.11^{\circ} \mathrm{N}, 99.24^{\circ} \mathrm{W}\right)$ and Cholula Puebla $(\mathrm{P})\left(19.1^{\circ} \mathrm{N}\right.$, $98.3^{\circ} \mathrm{W}$ ) (see Fig. 1). The data acquisition discussed in this work was made during the years 1992 and 1993, although Puebla station worked only some months of 1992. At each electroseismic station two time series were simultaneously recorded (NS and EW channels). Due to technical adjustments, two different sampling rates $(\Delta \mathrm{t}=4 \mathrm{~s}$ or $\Delta \mathrm{t}=2 \mathrm{~s})$ were used in different time intervals along the mentioned period (Yepez et al., 1995). Some problems were present in the data acquisition, like lacks of data and outliers, among others inherent to the storage process; therefore, in order to minimize these problems, a pre-processed procedure was applied. First, the small lacks were filled and the higher frequencies were removed by means of a moving average each two minutes ( 30 or 60 samples), because the seismic phenomenon is given in the ultra low frequencies range. The elimination of outliers was performed in order to have the same reference level, leading to more homogeneous data. Finally, the local tendencies of $(\Delta V / L)$ were removed by a detrending procedure with $\mu=0$. In Figs. 2, 3 and 4 some pre-processed time series are depicted. 

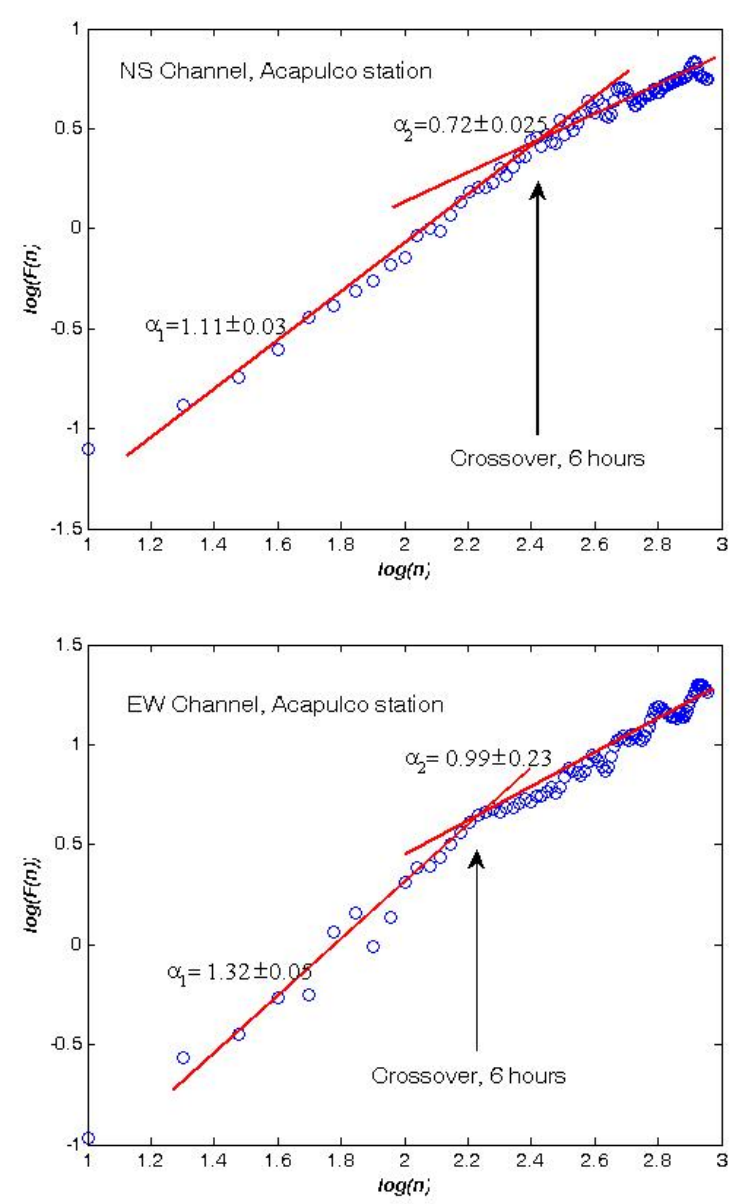

Fig. 5. Some examples of crossover observed in DFA of the Acapulco station time series. The crossover point corresponds to a lag of about $6 \mathrm{~h}$.

\section{Results}

The aim of this work is to establish a characterization of the electric self-potential fluctuations monitored at sites located on regions I, II and III showed in Fig. 1. The first considered region is located at the state of Puebla México, where we installed a station at $\mathrm{P}\left(19.1^{\circ} \mathrm{N}, 98.3^{\circ} \mathrm{W}\right)$. Gonzalez-Pomposo and Valdez-Gonzalez (1995) have reported seismicity measurements in this region, which is characterized by seisms with $\mathrm{M}_{c}<4$. The number of microseisms in this zone is in the order of one hundred per year. This microseismicity level is remarkably lesser than that of Guerrero state (region III) (Singh et al., 1983). In region III we installed the station $\mathrm{A}\left(16.85^{\circ} \mathrm{N}, 99.9^{\circ} \mathrm{W}\right)$. In this region are very common seisms with $\mathrm{M}>4$, which are quite infrequent in region $\mathrm{I}$. Both considered regions have similar surfaces (in the order of $3-6 \times 10^{4} \mathrm{~km}^{2}$ ). Region II corresponds to a zone of intermediate seismicity (Singh et al., 1983), where we installed the
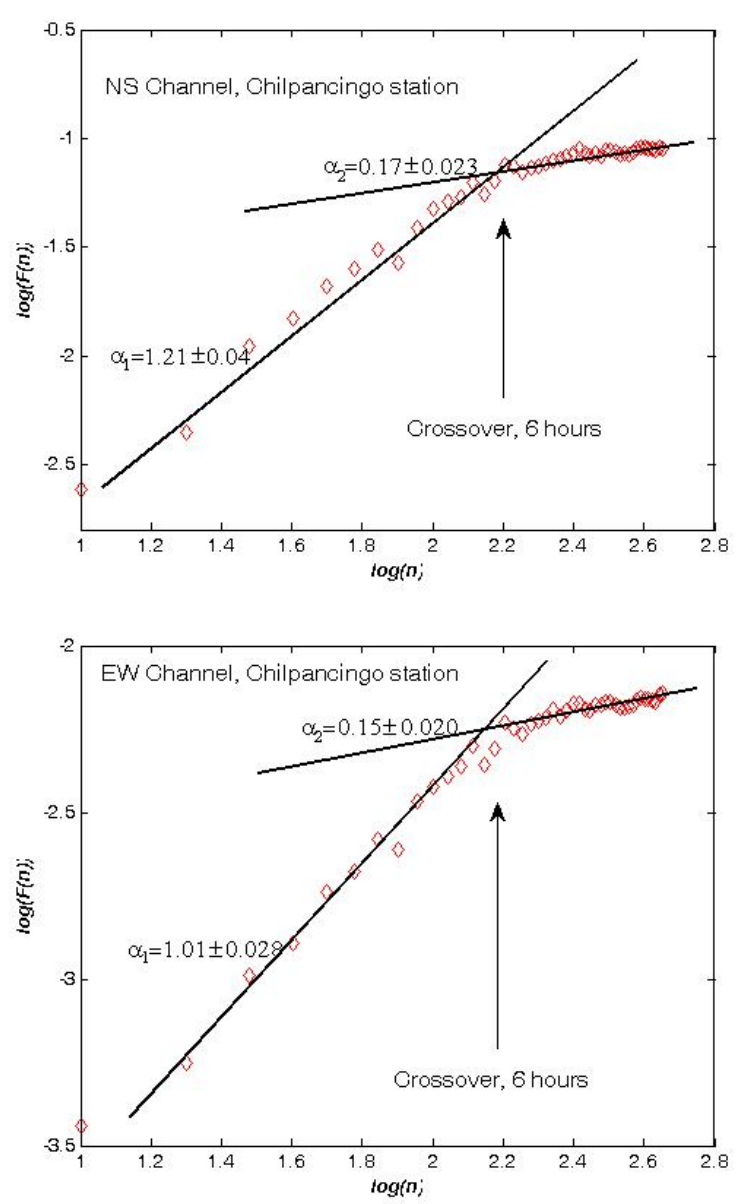

Fig. 6. Some examples of crossover observed in DFA of the Chilpancingo station time series. The crossover point corresponds to about a lag of $6 \mathrm{~h}$.

C station $\left(17.11^{\circ} \mathrm{N}, 99.24^{\circ} \mathrm{W}\right)$. We consider that the level of seismicity should be correlated with the natural electric activity. Nevertheless, the anthropogenic activity represents an important component of noise; therefore the methodology employed must have the capacity to distinguish the most important characteristics. The Detrended Fluctuation Analysis (DFA) and Higuchi algorithm were applied to data sets as those of Figs. 2, 3 and 4. We have chosen nonoverlapping time windows of five days (3600 points) to compute the fractal dimension $D$ (Eq. 2) and DFA exponents, $\alpha$, (Eq. 4).

In the case of the first method, DFA, Figs. 5 and 6 show representative $\log$ - $\log$ plots of $F(n)$ vs. $n$. For Acapulco station a crossover in the scaling exponent is observed in both channels, over short scales $\alpha_{1}$ is close to one (see Fig. 5). For Chilpancingo station, we observed a more accentuated crossover which reveals changes in the fractal dynamics organization (see Fig. 6). In this case, for short scales the exponent remains close to one but over large scales the $\alpha$ value 

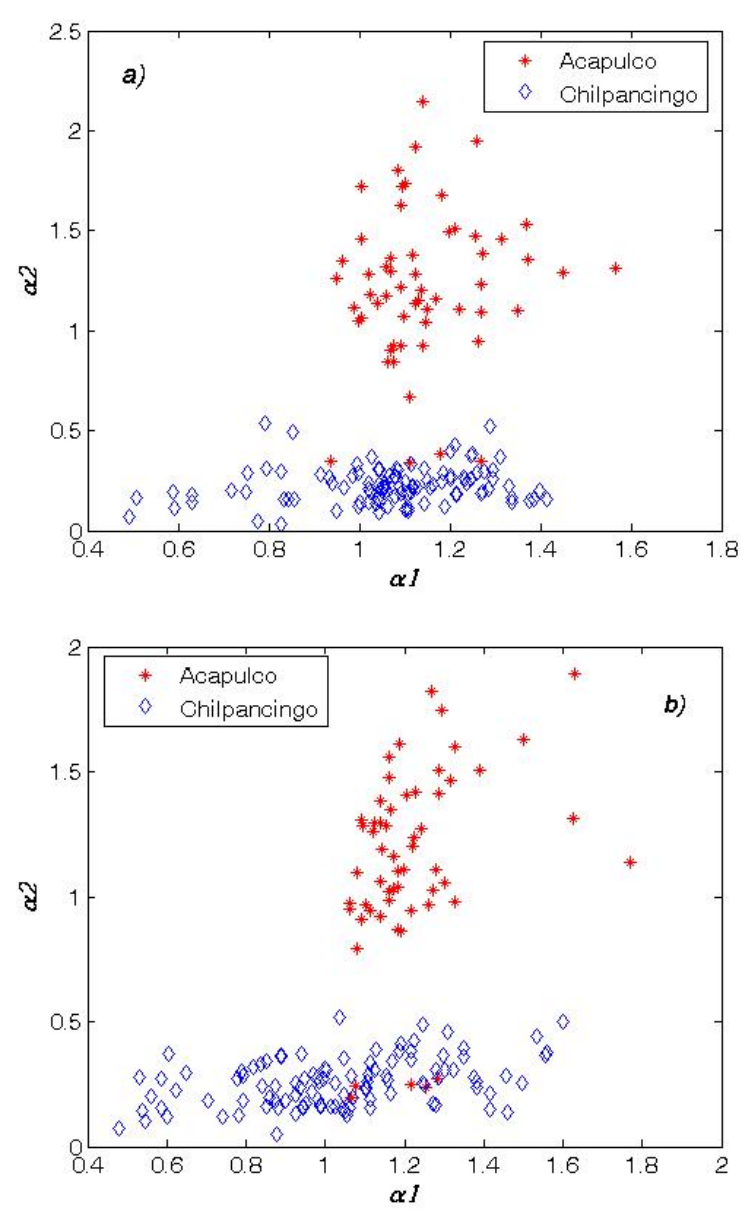

Fig. 7. Scatter plot of $\alpha_{2}$ vs. $\alpha_{1}$. A clear separation between Acapulco and Chilpancingo data is shown (a) NS Channel, (b) EW Channel.

is smaller than 0.5 , indicating antipersistent behavior. The pre-processed time series of Puebla (Fig. 4) had several important lacks of data such that it was not possible to calculate representative DFA exponents.

To go further inside in the comparison of our data sets, we constructed scatter plots of DFA exponents for Acapulco and Chilpancingo data, $\alpha_{1}$ vs. $\alpha_{2}$, and for both channels. Those are depicted in Fig. 7, $\alpha_{2}$ and $\alpha_{1}$ are the scaling exponents associated with large and short window sizes respectively. In this case we observe segregation between the points corresponding to the high level seismicity region (III) and the intermediate level seismicity region (II). The average values of $\alpha_{2}$ and $\alpha_{1}$ for both channels are $<\alpha_{2}>_{N S}=1.225 \pm 0.380,<\alpha_{1}>_{N S}=1.142 \pm 0.120$, $<\alpha_{2}>_{E W}=1.139 \pm 0.370,<\alpha_{1}>_{E W}=1.219 \pm 0.140$ for Acapulco respectively. For Chilpancigo station the average results are $<\alpha_{2}>_{N S}=0.227 \pm 0.090,<\alpha_{1}>_{N S}=1.064 \pm 0.190$, $<\alpha_{2}>_{E W}=0.260 \pm 0.090, \quad<\alpha_{1}>_{E W}=1.028 \pm 0.250$ respectively.

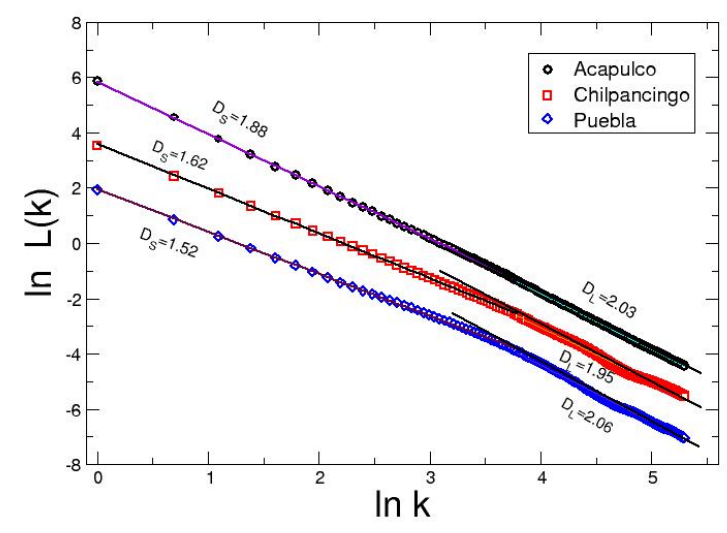

Fig. 8. $\log -\log$ plot of $<L(k)>$ vs. $k$ for representative cases of the three regions. A clear crossover is observed for Chilapancingo and Puebla data.

When Higuchi's algorithm was applied, a crossover is also observed in the log-log plots of $\langle L(k)>v s k$ (Eq. 2) and two fractal dimensions $D_{S}$ and $D_{L}$ can be determined. Here, $D_{S}$ and $D_{L}$ means fractal dimension of short and large scales. In Fig. 8 representative cases of the crossover behavior are described. For Acapulco data, a weak change in the scaling exponent value is observed whereas for Chilpancingo and Puebla a clear crossover is identified.

We notice that for Puebla data, we observed a more evident crossover, which indicates a clear separation in the fractal dimension of short and large scales. In Fig. 9, we present the scatter plot constructed with $D_{S}$ vs. $D_{L}$. For this case we also observe a reasonable segregation of points for the three regions considered. The average values $D_{S}$ and $D_{L}$ are: Chilpancingo $\left(<D_{S}>_{N S}=1.611 \pm 0.150,<D_{L}>_{N S}=2.019 \pm 0.044\right.$, $\left.<D_{S}>_{E W}=1.753 \pm 0.174,<D_{L}>_{E W}=2.043 \pm 0.040\right)$; Acapulco $\left(<D_{S}>_{N S}=1.912 \pm 0.033,<D_{L}>_{N S}=2.058 \pm 0.145\right.$, $\left.<D_{S}>_{E W}=1.859 \pm 0.043, \quad<D_{L}>_{E W}=2.063 \pm 0.017\right)$ and Puebla $\left(<D_{S}>_{N S}=1.299 \pm 0.152,<D_{L}>_{N S}=2.066 \pm 0.053\right.$, $\left.<D_{S}>_{E W}=1.347 \pm 0.120,<D_{L}>_{E W}=2.019 \pm 0.041\right)$.

\section{Discussion and concluding remarks}

Some authors (Telesca et al., 2001) have reported a good correlation between the dynamical mechanism governing the seismic and geoelectric phenomena. In the case of Telesca et al. (2001) the Hurst exponent of seismic sequences and the spectral power-law exponent of geoelectric time series at southern Italy were used to establish such a good correlation. We did not present a comparative study of geoelectric and seismic activities in the sense of Telesca et al. (2001) because at the present day we have not the seismic catalogues for the 

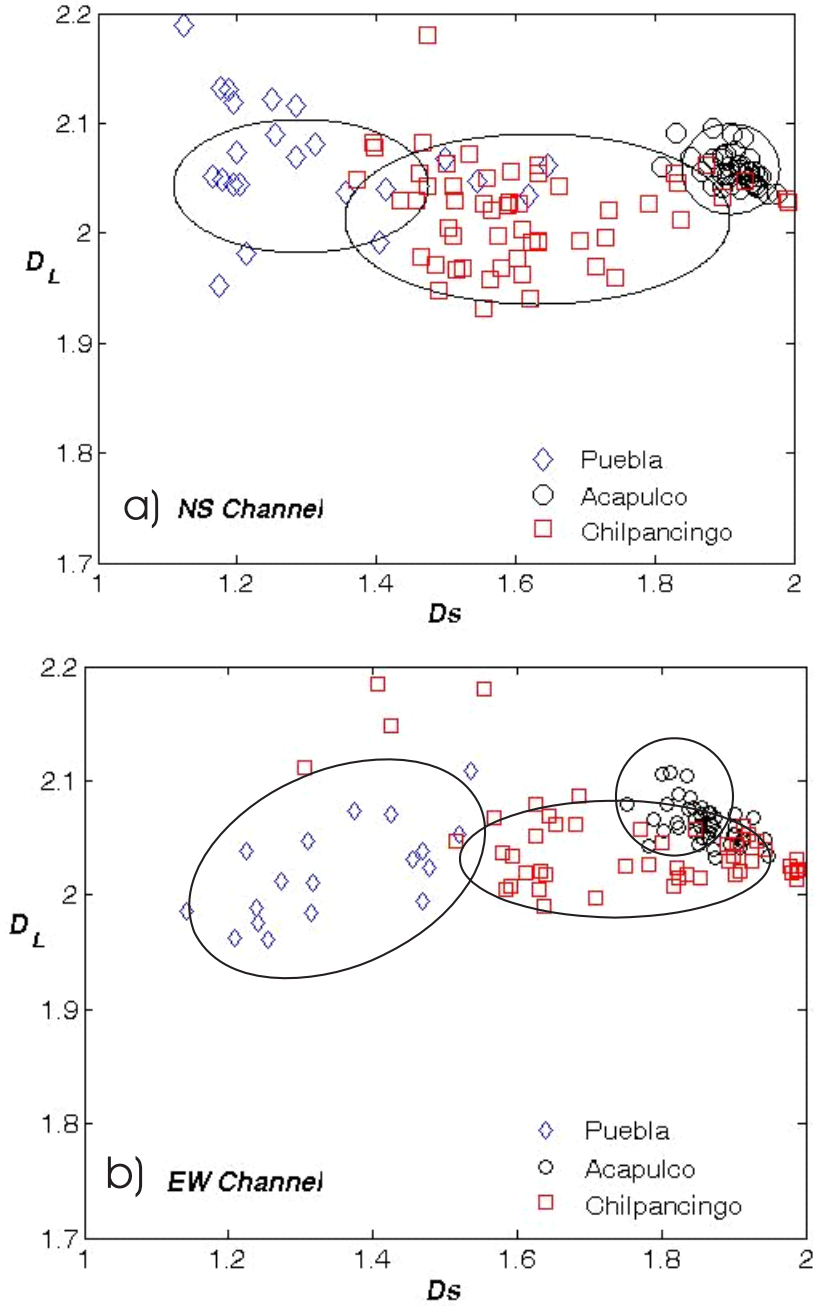

Fig. 9. Scatter plot of $D_{S}$ vs. $D_{L}$. In general, it is observed a separation between the corresponding points of the three regions considered. (a) NS Channel, (b) EW Channel.

monitored regions. In the present study the different levels of seismicity for three Mexican regions are taken as given information and then the difference in the geoelectric activity is explored through the DFA-method and the Higuchi fractal dimension, in Fig. 7 we depicted the DFA scatter plots of $\alpha_{1}$ vs. $\alpha_{2}$, for both Acapulco and Chilpancingo stations (NS and EW channels). A clear segregation between the points $\left(\alpha_{1}, \alpha_{2}\right)$ corresponding to the high seismicity region III, and those of the intermediate seismicity region II, is observed for both channels (NS and EW). Nevertheless the wide scattering of points pertaining to the both separated clouds we calculate the average of $\alpha_{1}$ and $\alpha_{2}$ (NS and EW). For the case of Chilpancingo station (region II), both, $<\alpha_{2}>$ in NS and EW directions result smaller than $\alpha=0.5$ (white noise) with an antipersistent behavior (Peng et al., 1995). For the case of $\alpha_{1}$, that is, the DFA-exponent for lags smaller than six hours, the
Chilpancingo data behaves as a good scale-free flicker noise $(\alpha \cong 1)$. In the case of the DFA - analysis of Acapulco station data, we find that the average DFA-exponents are reasonably near of $1 / f$ noise for both directions. Thus, within the DFA context the main difference between both data sets is that for lags larger than six hours Chilpancingo losses its $1 / f$ behavior becoming a series with near white noise behavior. By means of the Higuchi's analysis in Fig. 9 we depict the scatter plots of $D_{S}$ vs. $D_{L}$ for the three regions considered. We see that the three clouds of points $\left(D_{S}, D_{L}\right)$ corresponding to the three seismic regions are approximately segregated. Regardless the wide scattering of these points, we calculated their average values obtaining the numerical results mentioned in the previous section. We observe that under this analysis all the fractal dimensions for large lags result approximately uncorrelated white noise. For short scales Acapulco is near $1 / f$ noise and Chilpancingo and Puebla are near Brownian noise (short-range correlations). For short scales, in the case of Puebla station we obtained the Higuchi dimensions given in Sect. 4, which by means of the so-called Berry's equation, $D=\frac{5-\beta}{2}$ (Berry, 1979; Turcotte, 1992), give the spectral exponents $\beta_{S, N S}=3.402$ and $\beta_{S, E W}=2.306$ representing a nonstationary series with strong persistence (Malamud and Turcotte, 2001). In summary, although both methods produce wide scattering of points, one can qualitatively see that geoelectric data for the three regions have a reasonable separation in both the DFA and Higuchi scatter plots. Thus, under a first approximation we can assert that the global differences in seismicity levels are translated into differences in global geoelectric activity. We must remark that the present analysis is only a first approximation because as it is well known, Earth has a very rough electrical conductivity structure and scaling exponents possibly provide a measure of heterogeneity in the distribution of the underlying electrical conductivity. Evidently our approach does not permit to distinguish any particular geological structure as major faults for example. However, other geoelectric approaches (Makris et al., 1999; Eftaxias et al., 2002; Balasis et al., 2002; Balasis et al., 2005; Varotsos et al., 1996) address the problem of linking geoelectric signals with the geoelectrical structure of the area that hosts the stations as well as the regional structure between the station and the seismic focal area.

Acknowledgements. E. L. Flores-Márquez thanks the Universidad Autonoma Metropolitana for the sabbatical financial support and PAPIIT-DGIA-UNAM (Mxico), research project number IN102906-2. FAB and LGV thank to COFAA and EDI-IPN for partial financial support. ARR thanks to Departamento de Ciencias Básicas-UAM for financial support.

Edited by: P. F. Biagi

Reviewed by: 3 anonymous referees 


\section{References}

Abe, S. and Suzuki, N.: Scale-invariant statistics of period in directed earthquake network, Eur. Phys. J. B., 44, 115-117, 2005.

Angulo-Brown, F., Ramirez-Guzman, A. H., Yepez, E., RudolfNavarro, A., and Pavia-Miller, C. G.: Fractal geometry and seismicity in the Mexican subduction zone, Geofis. Int., 37, 29-33, 1998.

Balasis, G., Bogris, N., and Eftaxias, K.: Magnetovariatonal and Magnetotelluric study of Ioannina region sensitive to Seismic Electric Signals (SES), II J. Atmos. Electricity, 22, 139-164, 2002.

Balasis, G., Bedrosian, P., and Eftaxias, K.: A magnetotelluric study of the sensitivity of an area to seismoelectric signals, Nat. Hazards Earth Syst. Sci., 5, 931-946, 2005,

http://www.nat-hazards-earth-syst-sci.net/5/931/2005/.

Berry, M. V.: Difractals, Phys. A Math. Gen., 12, 207-220, 1979.

Cervantes de la Torre F., Ramirez-Rojas, A., Pavia-Miller, C. G., Angulo-Brown, F., Yepez, E., and Peralta, J. A.: A comparison between spectral and fractal methods in electrotelluric time series, Rev. Mex Fis., 45, 298-302, 1999.

Dobrovolsky, I. P., Gershenzon, N. I., and Gokhberg, M. B.: Theory of electrokinetic effects occurring at the final stage in the preparation of a tectonic earthquake, Phys. Earth Planet. Int., 57, 144-156, 1989.

Eftaxias, K., Rokityansky, I., Bogris, N., Balasis, G., and Varotsos, P.: Magnetovariatonal and Magnetotelluric study of Ioannina region sensitive to Seismic Electric Signals (SES), I J. Atmos. Electricity, 22, 113-137, 2002.

Eftaxias, K., Kapiris, P., Polygiannakis, J., Kopanas, J., Peratzakis, A., Skountzos, P., and Jaggard, D.: Review and a Model of Pre-Seismic electromagnetic emissions in terms of fractal electrodynamics, Fractals, 12, 243-273, 2004.

Freund, F.: Seismic electric signals (SES) and the conductivity structure of the crust, Seismoelctromagnetics: Lithosphere,Atmosphere-Ionosphere Coupling, edited by: Hayakawa, M. and Molchanov, O. A., 141-152, Terrapub Tokyo, 2002.

Gonzalez-Pomposo, G. J. and Valdes-Gonzalez, C.: Seismicity of the state of Puebla, México 1986-1989, Geofís. Int., 34, 221231, 1995.

Gotoh, K., Hayakawa, M., and Smirnova, N.: Fractal analysis of the ULF geomagnetic data obtained at Izu Peninsula, Japan in relation to the nearby earthquake swarm of June-August 2000, Nat. Haz. Earth. Syst. Sci., 3, 229-236, 2003.

Hayakawa, M., Ito, T., and Smirnova, N.: Fractal analysis of ULF geomagnetic data associated with the Guam earthquake on $8 \mathrm{Au}-$ gust 1993, Geophys Res. Lett., 26, 2797-2800, 1999.

Hayakawa, M. and Ito, T.: ULF electromagnetic precursors for an earthquake at Biak, Indonesia on 17 February 1996, Geophys. Res. Lett., 27, 1531-1534, 2000.

Higuchi T.: Approach to an irregular time series on basis of the fractal theory, Physica D., 31, 277-283, 1988.

Higuchi T.: Relationship between the fractal dimension and the power law index for a time series: a numerical investigation, Physica D., 46, 254-264, 1990.

Malamud, B. D. and Turcotte D. L.: Self-Affine Time Series: I Generation and Analyses, Sixth Workshop on Non-Linear Dynamics and Earthquake Prediction, H4.SMR/1330-22, 2001.

Makris, J., Bogris, N. and Eftaxias, K.: A new approach in the determination of characteristic directions of the geoelectric structure using Mohr circles, Earth Planet Space, 1059-1065, 1999.

Nitsan, U.: Electromagnetic emission accompanying fracture of quartz-bearing rocks, Geophys. Res. Lett., 4, 333-336, 1977.

Nikolopoulos, S., Kapiris, P., Karamanos, N., and Eftaxias, K.: A unified approach of catastrophic events, Nat. Hazards Earth Syst. Sci., 4, 615-631, 2004, http://www.nat-hazards-earth-syst-sci.net/4/615/2004/.

Peng, C. K., Budyrev, S. V., Havlin, S., Simons, M., Stanley, H. E., and Goldberger, A. L.: Mosaic organization of DNA nucleotides, Phys. Rev. E, 49, 2, 1685-1689, 1994.

Peng, C. K., Havlin, S., Stanley, H. E., and Goldberger, A. L.: Quantification of scaling exponents and crossover phenomena in non stationary hearbeat time series, Chaos, 5, 1, 82-87, 1995.

Ramirez-Guzman, A.: Geometria fractal y sismicidad: dos casos en el suroccidente y sureste de Mexico, ESFM-IPN. Msc. Thesis (in Spanish), 1996.

Ramirez-Rojas, A., Pavia-Miller, C. G., and Angulo-Brown, F.: Statistical behavior of the spectral exponent and the correlation time of electric self-potential time series associated to the Ms=7.4 September 14, 1995 earthquake in Mexico, Phys. Chem. Earth, 29(4-9), 305-312, 2004.

Ramirez-Rojas, A., Muñoz-Diosdado A., Pavia-Miller, C. G., and Angulo-Brown, F.: Spectral and multifractal study of electroseismic time series associated to the $\mathrm{M}_{w}=6.5$ earthquake of 24 October 1993 in Mexico, Nat. Hazards Earth Syst. Sci., 4, 703709, 2004, http://www.nat-hazards-earth-syst-sci.net/4/703/2004/.

Singh, S. K., Rodriguez, M., and Esteva, L.: Statistics of small earthquakes and frequency of occurrence of large earthquakes along the Mexican subduction zone, Bull. Seism. Soc. Am., 73, 1779-1796, 1983.

Smirnova, N., Hayakawa, M., Gotoh, K., and Volobuev, D.: Scaling characteristics of ULF geomagnetic fields at the Guam seismoactive area and their dynamics in relation to the earthquake, Nat. Hazards Earth Syst. Sci., 1, 119-126, 2001, http://www.nat-hazards-earth-syst-sci.net/1/119/2001/.

Sobolev, G. A., Semerchan, A. A., Salov, B. G., Spetzler, H. A., Sondergeld, C. H., Badanov, V. N., Kol'tsov, A. V., Los', V. F., Nasimov, R. M., Ponomarev, A. V., Stakhovski, T. R., Teren'ev, V. A., and Turetskii, I. M.: Precursor of destruction of a large rocks sample, Izvetsiya. Earth Phys., 18, 572-580, 1982.

Stacey, F. D.: The seismomagnetic Effect, Pure Appl. Geophys., 58, 5-22, 1964.

Stacey, F. D. and Johnson, M. J. S.: Theory of the piezomagnetic effect in Titanomagnetic-Bearing Rocks, Pure Appl. Geophys., 97, 146-155, 1972.

Telesca, L., Cuomo, V., Lapenna, V., and Macchiato, M.: A new approach to investigate the correlation between geoelectrical time fluctuations and earthquakes in a seismic area of southern Italy, Geophys. Res. Lett., 28, 4375-4378, 2001.

Telesca, L., Colangelo, G., Lapenna, V., and Macchiato, M.: Monofractal and multifractal characterization of geoelectrical signals measured in southern Italy, Chaos Solitons Fractals, 18, 385-399, 2003.

Telesca, L., Lapenna, V., and Macciato, M.: Multifractal fluctuation in earthquake related geoelectrical signals, New J. Phys., 7, 214, doi:10.1088/1367-2630/7/1/214, 2005a.

Telesca, L., Colangelo, G., and Lapenna, V.: Multifractal variability in geoelectrical signals and correlation with seismicity: a study 
case in southern Italy, Nat. Hazards Earth Syst. Sci., 5, 673-677, 2005b.

Telesca, L. and Lapenna, V.: Measuring multifractality in seismic sequences, Tectonophysics, 423, 115-123, 2006.

Telesca, L., Balasco, M., and Lapenna, V.: Investigating the timecorrelation properties in self-potential signals recorded in a seismic area of Irpinia, southern Italy, Chaos Solitons Fractals, 32, 199-211, 2007.

Turcotte, D. L.: Fractals and Chaos in Geology and Geophysics, Cambridge University Press, 221, 1992.

Vallianatos F., Triantis, D., Tzonis, A., Anastiasadis, C., and Staurakos, I.: Phys. Chem. Earth, 29(4-9), 339-351, 2004.

Varotsos, P. and Alexopoulos, K.: Physical properties of the variations of the electric field of the Earth preceding earthquakes, I. Tectonophysics (110), Elsevier Science Publishers, 1984a.

Varotsos, P. and Alexopoulos, K.: Physical properties of the variations of the electric field of the Earth preceding earthquakes, II. Tectonophysics (110), Elsevier Science Publishers, 1984b.
Varotsos, P., Lazaridou, M., Eftaxias, K., Antonopoulos, G., Makris, J., and Kopanas, J.: Short term earthquake prediction in Greece by seismic electric signals, A critical Review of VAN, (Editor: Sir James Lighthill), 29-76, Word Scientific, 1996.

Varotsos, P. A., Sarlis, N. V., and Skordas, E. S.: Long-range correlations in the electric signals that precede rupture, Phys. Rev. E, 66, 011902, 2002.

Varotsos, P. A., Sarlis, N. V., and Skordas, E. S.: Long-range correlations in the electric signals that precede rupture: Further investigations, Phys. Rev. E, 67, 021109, 2003a.

Varotsos, P. A., Sarlis, N. V., and Skordas, E. S.: Attempt to distinguish electric signals of a dichotomous nature, Phys. Rev. E, 68, 031106, 2003b.

Yepez, E., Angulo-Brown, F., Peralta, J. A., Pavia-Miller, C. G., and Gonzalez-Santos, G.: Electric fields patterns as seismic precursors, Geophys. Res. Lett. 22, 3087-3090, 1995. 